\title{
Towards a Science of Checklists
}

\author{
Hajo A. Reijers \\ Vrije Universiteit Amsterdam \\ $\underline{\text { h.a.reijers@vu.nl }}$
}

\author{
Henrik Leopold \\ Vrije Universiteit Amsterdam \\ $\underline{\text { h.leopold@,vu.nl }}$
}

\author{
Jan Recker \\ Queensland University of \\ Technology \\ j.recker@qut.edu.au
}

\begin{abstract}
Checklists are in use in many work domains, including aviation, manufacturing, quality control, and healthcare. Despite their adoption, the literature shows both breadth and persistence of problems with the organizational usage of checklists. In this paper, we conduct a structured literature survey to analyze checklists from the perspective of informational artifacts. Our contribution is a respective conceptualization of checklists and a rigorous analysis of their problems. As we will argue, these insights help to consider how the capabilities of IT systems can be leveraged to improve checklists and address their problematic aspects. We present our work as a basis for IT-oriented research into a relevant yet underexamined information practice in organizational work routines.
\end{abstract}

\section{Introduction}

Checklists are pervasive. From the simple shopping lists in daily life to comprehensive pre-flight checklists in aviation, people use these devices to guide and verify their actions. The use of checklists has also shown to be highly beneficial. Probably the most wellknown example is the surgical checklist that was developed on basis of guidelines by the World Health Organization, which led to halving the mortality rates in patient populations of the hospitals who started applying it [1]. Because of their success to reduce the likelihood of human error to occur under daily work conditions, checklists have also become highly pervasive in aviation, where they are a mandatory part of practice [2].

Despite this positive connection between the use of checklists and organizational performance, they do not come without problems. For example, "checklist fatigue" has become a well-observed phenomenon, whereby the overwhelming number of available or required checklists becomes a hindrance rather than an aid [3]. Also, checklist adoption varies drastically in spite of their demonstrated benefits [4].
While researchers and practitioners have been working on addressing these and other checklist issues, two characteristics of the proposed solutions are worth pointing out. First of all, the design and implementation of checklists has mostly been a domain-centered approach: aviation engineers work on aviation checklists, medical professionals on medical checklists, and so forth. Secondly, none of the solutions we have seen are coupled to something that could be construed fundamental properties of checklists. Instead, they focus on organizational measures to improve the acceptance and use of checklists or deal with their surface aspects only. These limitations have led to calls for a "science of checklists" [5] - yet, with little follow-up to date.

In this paper, we wish to shed a new, information systems-oriented perspective on checklists. Specifically, we propose to view and analyze checklists as principally informational artifacts that guide work. This allows for a reflection on IT system attributes that can be exploited to reshape checklists and, by doing so, overcome pertinent issues with their usage. The upshot is that checklists may become applicable in even wider societal settings than is currently the case, with all their associated benefits.

Following a structured literature review, the main contributions of this work are (1) a conceptualization of checklists as informational artifacts and (2) a rigorous analysis of existing checklist problems. We will build on these to argue that an IT-driven overhaul of checklists may both be feasible and attractive.

We proceed as follows. First, we introduce the notion of a checklist in Section 2. In Section 3, we explain the methodological details of our structured literature survey. Section 4 then presents our conceptualization of checklists as informational artifacts. In Section 5, we provide a comprehensive overview of the problems with checklist usage, as well as the known strategies to deal with these. Our proposals to address pertinent issues are presented in Section 6. Section 7 concludes this paper. 


\section{What is a Checklist?}

A lemma in the Merriam-Webster dictionary describes a checklist as a "list of things to be checked or done". This definition captures the supportive nature of a checklist to "tick off" work that has been carried out and to serve as a reminder of what is still left to do. Note how the concept of a "to-do list" specifically taps into the latter aspect of a checklist. This is why we will consider it as being subsumed by the wider checklist notion.

Another notion worth mentioning is that of the "timeout" aspect of checklists, which is popular in the healthcare domain. It refers to the particular pause that allows a team to go through a relevant checklist just before an important procedure. Although it is colloquially used as a synonym for a checklist, we think it is preferable to distinguish the list from the interruption of work.

For the sake of clarity, we follow [2] for our definition of a checklist: "A checklist is typically a list of action items or criteria arranged in a systematic manner, allowing the user to record the presence/absence of the individual items listed to ensure that all are considered or completed".

As an example of a checklist, consider the Surgical Safety Checklist from the World Health Organization (see Figure 1). This checklist is intended to support surgical personnel to guarantee the safety of patients before, during, and after a surgery. Already a first glance reveals that this checklist is more than a simple collection of check boxes. We will be using this example to explain our conceptualization of checklists below.

\section{Methodology}

To be able to conceptualize checklists as informational artifacts and to develop a comprehensive overview of the issues that are associated with them, we carried out a structured literature survey. Our goal was to aggregate data on a narrow scope, viz., concepts and issues with checklists reported in academic literature. Therefore, our literature review is a type of qualitative systematic review [26]. We carried out the review as follows.

Since checklists are used in a wide variety of domains, we did not opt for any domain-specific libraries or databases. Instead, we decided to use Google Scholar, which can be considered as the most comprehensive search engine for the academic literature at this point, with coverage of all scientific domains. As search terms we used the exact phrases "checklist characteristics", "characteristics of checklists", "problem with a checklist", "problems with a checklist", "problem with checklists", and "problems with checklists". In this way, we covered both literature discussing the concepts of checklists as well as the problems that occurred with their usage.

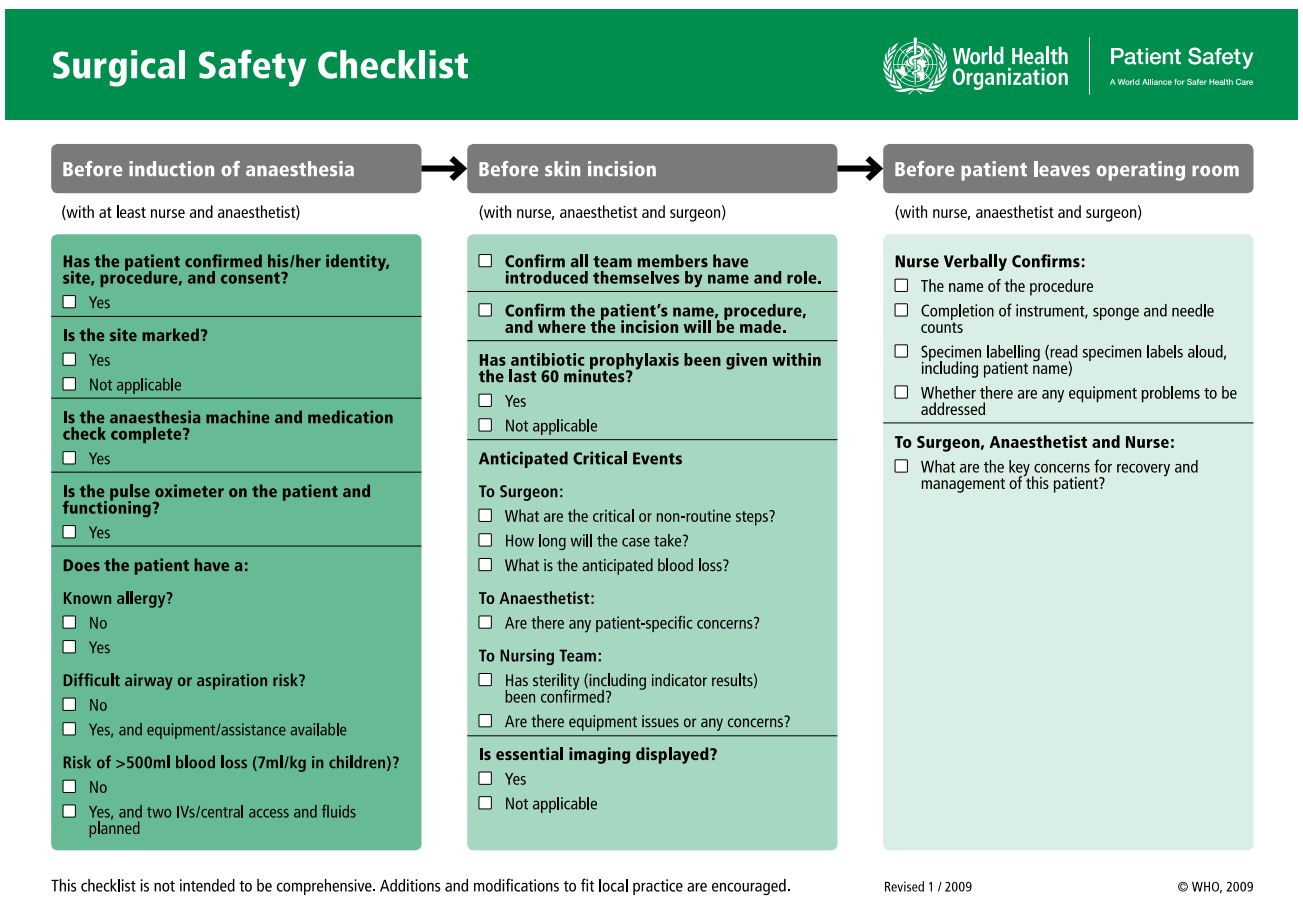

Figure 1: Example of a checklist [6] 
Note that more generic search terms such as "checklist" or "checklist problem" turned out to be not selective enough: They often led to papers that reported on the actual use of checklists to diagnose and solve a range of problems, i.e. they captured an operational instead of a reflective perspective on checklists and their management.

Our search led to the identification of 101 academic papers of which 56 papers could be retrieved. All of these papers were read in full to identify concepts and issues that seemed potentially applicable to more than a single checklist. To complement this broad-brush procedure, we conducted a qualitative search, which we initiated on the basis of a recent publication in Nature: "The Trouble With Checklists" [27]. We analyzed this paper for additional concepts and problem types and traced all the cited references. We screened these papers for relevance and added them to our pool in case we would expect the identification of additional recurring checklist concepts and issues from them. We repeated this procedure for all references of newly found papers until the search was exhausted. For all the papers that were added in this way, we also screened the papers citing these works themselves, in this way implementing a "snowballing" procedure. This additional search procedure led to the identification of 31 further papers.

In a final step, we iteratively applied open coding to identify and group concepts and issues. The result was a checklist conceptualization based on their scope and properties as well as a number of reoccurring issue types. In what follows, we will first introduce the conceptualization of checklists.

\section{Conceptualization of Checklists as Informational Artifacts}

The key postulation behind our work is that checklists are a type of informational artifact that conceptualize activities and decisions in work routines. Informational artifacts encapsulate, abstract, and represent all relevant information about some realworld phenomena in a single abstraction. This assumption suggests that the essential purpose of checklists is twofold: (1) describe work routines (which we call the aspect of representation) and (2) guide decisions and tasks within such routines (the aspect of prescription). The nature of checklists is then that they are a type of conceptual model [7] that provide a purposeful and relevant representation of a particular real-world domain (the aspect of abstraction).

This assumption is useful for delineating properties of checklists as information artifacts in two ways. First, because it implies that the nature and purpose of checklists is similar to other approaches used for conceptualizing activities and decisions carried out in some real-world domain such as process models [8][9], routine networks [10], or state machines [11]. This will be fruitful for identifying IT strategies available for this artifacts and applying them to checklists, as we will illustrate below. Second, because it allows developing an understanding of relevant properties in general [12] that describe types of checklists, which is useful to discriminate specific kinds of checklists that are or may be in use. We return to this issue as well.

To offer a first conceptualization of the spectrum of kinds of checklists, we identify seven properties (see Table 1). Five of them relate to the entire checklist, while two relate to its constituting elements in particular, i.e. the checklist items.

Table 1: Properties of checklists

\begin{tabular}{|l|l|l|}
\hline Scope & Property & Selected values \\
\hline \multirow{4}{*}{$\begin{array}{l}\text { Entire } \\
\text { Checklist }\end{array}$} & Representation & $\begin{array}{l}\text { Paper, poster, } \\
\text { mechanical, electronic, } \\
\text { vocal }\end{array}$ \\
\cline { 2 - 3 } & Prescriptiveness & $\begin{array}{l}\text { Do-list, call-do } \\
\text { response }\end{array}$ \\
\cline { 2 - 3 } & Abstraction & $\begin{array}{l}\text { System engineering, } \\
\text { human performance }\end{array}$ \\
\cline { 2 - 3 } & Audience & $\begin{array}{l}\text { Normal, abnormal, } \\
\text { emergency }\end{array}$ \\
\cline { 2 - 3 } & Type & Individual, group \\
\hline \multirow{4}{*}{$\begin{array}{l}\text { Checklist } \\
\text { items }\end{array}$} & $\begin{array}{l}\text { Behavioral } \\
\text { relation }\end{array}$ & $\begin{array}{l}\text { Check, score, multiple } \\
\text { choice, branched, } \\
\text { interrogative }\end{array}$ \\
\cline { 2 - 3 } & $\begin{array}{l}\text { Arbitrary, strongly } \\
\text { sequential, weakly } \\
\text { sequential, parallel }\end{array}$ \\
\hline
\end{tabular}

\subsection{Properties of the Entire Checklist}

The first checklist property concerns the aspect of representation of checklists, that is, the question of how a work routine, its activities and decisions, are conceptualized in a checklist. The most common and also simplest representation of a checklist is a paperbased checklist. The advantage of a paper-based checklist is its low technical complexity and high reliability [13]. Furthermore, it is portable and easily reproducible. An alternative paper-based type of checklist is a poster checklist [14]. In comparison to a regular paper-based checklist, it has the advantage that it is visible to a larger group of people. In particular, in a surgical setting this may represent an important characteristic of the checklist. Non-paper-based 
representations of checklists include mechanical, electronic, and vocal checklists. Mechanical checklists consist of a panel composed of several plastic slides moving over a list of checklist items [15]. One important feature of mechanical checklists is that they only show the non-accomplished items. They are, for instance, used in aviation in the context of takeoff and landing. The term electronic checklist or automated checklist applies to any checklist that is shown on a digital display, which assumes that it is digitally represented in some way. Electronic checklists are marked using a cursor and have the advantage of dynamic updates and a wide range of visualization opportunities. The biggest advantage of electronic checklists, however, is the opportunity of an associated system to technically check whether a task was actually performed [13]. Especially in critical domains such as aviation, this can be an important feature. Vocal checklists are typically considered as a special type of electronic checklist. In essence, a vocal checklist is technical unit that "reads" the checklist items to the user [16]. The user confirms the accomplishment of a task by pushing a button. Recent research indicated that such audible checklists can lead to a decrease of in-flight errors by pilots [17]. Relating the aspect of representation to the checklist from Figure 1, we observe a paper-based checklist. However, it is also worth noticing that this checklist instructs to read certain aspects out loud ("read specimen labels aloud").

The second checklist property concerns the aspect of prescription of checklists, that is, the question of which type of guidance is provided through the checklist to a user. We found two dominant checklist prescriptions in use: call-do response and do-verify [13] The call-do-response method, often also called dolist or challenge-do-response checklist, follows a stepby-step "cookbook" approach [16]. When using the list, a user first calls an item from the list, then performs the action, and finally verifies the successful accomplishment of that action. This method is particularly effective when different persons are involved. One person could then perform the action, while the other takes care of the verification [13]. A typical application scenario for a call-do-response checklist is a surgical setting where safety-relevant aspects can be cross-checked [18]. A checklist that follows the do-verify method has the character of a backup. Users first rely on their memory to perform a number of required tasks. Then, they use the checklist to verify that each task has been accomplished successfully. As an example, consider a pilot, who first configures the aircraft according to memory and then uses the checklist to verify that all configurations were correctly set [16]. Note that the checklist from Figure 1 is a typical call-do-response checklist. The users of this checklist are supposed to go through the list step by step and verify for each item that has been completed successfully.

The third checklist property, scope, concerns the coverage aspect, that is, to what extent the checklist covers the entire list of required tasks. We distinguish two approaches to coverage: the system engineering approach and the human performance approach. The main rationale behind the systems engineering approach is that all items related to a task should be checked. As a result, the user potentially faces a long checklist with an extensive number of items. The human performance approach, by contrast, emphasizes that a detailed checklist is no guarantee for preventing human failure. According to the human performance approach, an extensive checklist rather carries the risk that users will fail to use it correctly or deny to use it altogether [13]. It can be seen that the checklist from Figure 1 represents an example of the human performance approach. It actually explicitly emphasizes this approach below the checklist: "This checklist is not intended to be comprehensive".

The forth checklist property concerns the aspect of abstraction, that is, the question which properties of the phenomena in the real-world domain are explicated through the checklist. Here we distinguish normal operations (checklists that capture routines how they should work in most cases), non-normal (checklists that capture escalations such as workarounds [19]), and emergency checklists (checklists that only capture singular routines such as disaster management or others). The checklist from Figure 1 illustrates that this distinction is not always and necessarily sharp. While it is intended for normal operations, it also encourages anticipating critical events (see the middle column).

The fifth checklist property concerns the aspect of audience, that is, whether the checklist is meant to be used by an individual or by a group of people. An example for a checklist that typically is meant to be used by two people is the previously introduced challenge-do-response checklist [5]. The checklist from Figure 1 illustrates this nicely. The target group of this checklist includes several roles including a nurse, an anesthesiologist, and a surgeon. For each of the three columns, the addressed roles are explicitly mentioned. In the right-hand column, we can even find specific instructions how their joint use of the checklist is meant to take place: "Nurse verbally confirms".

\subsection{Properties of the Checklist Items}

While the previously discussed properties relate to the entire checklist, there also two key characteristics 
of a checklist that particularly relate to the items that compose the checklist.

The first property concerns the type of a checklist item. While the term "checklist" suggests that items simply need to be ticked off, checklist items are, in fact, much more multi-faceted. We distinguish between five item types: check, score, multiple-choice, interrogative, and branched. The check type represents the simplest form of a checklist item: It consists of a task or a goal that has to be accomplished. Typically, the item is ticked off once the associated task has been performed. However, especially in aviation, check items are not marked [13]. Checklist items of the score type provide the user with the possibility to assign a score to an item (e.g., from 1 to 10). These items are often used in checklists for evaluation purposes. An example is the trauma checklist from [20], which consists of a number of symptoms that are scored from 0 to 3 . Items of the multiple-choice type offer the user with several response possibilities [21]. Hence, they are used in situations where the responses or outcomes are already known upfront. The simplest form of a multiple choice item is the yes/no item. For instance, a checklist that consists of 23 yes/no items to diagnose autism among toddlers is described in [22]. However, multiple-choice items may also relate to more specific outcomes. When several multiple-choice items are combined in such a way that the outcome of one choice leads to another, we refer to this as a branched item [21]. A branched item explicitly shows the dependencies between items and, therefore, can be beneficial for representing nested choices. Checklist items that are interrogative require the user to provide feedback. Thus, they typically consist of a question and an empty field [21]. Such items are particularly useful when the set of answers cannot be anticipated. The checklist from Figure 1 illustrates that a single checklist can combine several item types. We observe a mix of check items ("Has the patient confirmed his/her identity, site, procedure, and consent?"), multiple choice items ("Is the site marked?"), and interrogative items ("What are the concerns for recovery and management of this patient?").

The second property concerns the behavioral relation between checklist items, that is, in which order the tasks related to two items have to be carried out. We distinguish between four types of behavioral relations: arbitrary, strongly sequential, weakly sequential, and parallel. An arbitrary relation between two items defines that the completion order of two items is of no importance [23]. The main purpose of checklists with arbitrary items is therefore to serve as a mnemonic device. When two checklist items are in a strongly sequential relationship, the order between these items has to be preserved to obtain valid outcomes. As an example, consider the case of a preflight checklist, where the accuracy of certain instruments depends on zeroing their settings in the first place [23]. It is important to note that a sequential relationship can also lead to the repetition of an item. Such iterations are necessary when problems or discoveries from later checklist items require the reconsideration of earlier ones [24]. A weakly sequential relationship between two items specifies that the completion order is only important from a psychological or efficiency point of view. It may, for instance, be beneficial to ask the user to carry out cognitively demanding tasks first, such that they are accomplished at the required level of quality [23]. While it is not as common as the previously described relations, two checklist items can also be in a parallel relationship. Such a situation occurs when a second item has to be completed while the realization of the first one is still in progress. An example of a parallel relationship can be found in the approach and landing checklist for aircrafts [25]. Among others, it requires the pilot to gear down while also taking care of the landing flaps. Since it might be not sufficient to wait for the first item to be completed, both tasks are associated with items that are in a parallel relationship. In practice, the combination of the above described relationships takes place in different ways. For instance, the checklist from Figure 1 combines strongly sequential order with weakly sequential order items. The three columns clearly need to be executed from left to right. The checklist even further specifies the point of time by stating "before induction of anesthesia", "before skin incision", and "before patient leaves operating room". Within each column, the order is partially strongly and partially weakly sequential. For instance, the confirmation of the patient's identity is clearly supposed to take place before the site is marked. The order in which allergies of aspiration risk are determined is certainly of less importance. However, going from top to bottom is the intended mode of use.

The above discussion illustrates the power but also the complexity of the checklist as an informational artifact. It is thus not surprising that practitioners face different types of problems with checklists. We take a detailed look at these in the next section.

\section{Checklists Problems and Solutions}

As a result of our literature survey, we derived 71 checklist issues, which we grouped into 21 recurring issue types. In this section, we introduce these recurring problems with checklist use, as well as the state-of-the-art strategies advocated to address these. 
Table 2: Issues with checklists

\begin{tabular}{|c|c|c|c|}
\hline Category & Problem & Citations & Related Checklist Property \\
\hline \multirow{2}{*}{$\begin{array}{l}\text { Checklist } \\
\text { Design }\end{array}$} & Measurement problems with items & 14 & Checklist item: Type \\
\hline & $\begin{array}{l}\text { Difficulty to come up with a standardized } \\
\text { version }\end{array}$ & 2 & Checklist: Representation, Scope, and Abstraction \\
\hline \multirow[t]{12}{*}{ Operational Use } & Checklist not sensitive to context or case & 19 & Checklist: Abstraction \\
\hline & Non-compliance & 14 & Checklist: Prescriptiveness \\
\hline & "Checklist fatigue" & 12 & Checklist: Prescriptiveness, Scope \\
\hline & Reliant on human judgment & 10 & $\begin{array}{l}\text { Checklist: Representation, } \\
\text { Prescriptiveness }\end{array}$ \\
\hline & Poor integration with existing process & 5 & Checklist: Representation, Scope \\
\hline & Cognitive issues & 4 & Checklist: Representation, Scope, and Abstraction \\
\hline & $\begin{array}{l}\text { Difficult to read status/ receive feedback } \\
\text { from checklist }\end{array}$ & 4 & $\begin{array}{l}\text { Checklist: Representation, } \\
\text { Abstraction, and Prescriptiveness }\end{array}$ \\
\hline & No predictive/prescriptive power & 3 & Checklist: Prescriptiveness \\
\hline & Duplication of tasks in existing process & 3 & Checklist item: Behavioral relation \\
\hline & Difficulty to deal with exceptions & 3 & Checklist: Abstraction \\
\hline & Use highly susceptible to production pressure & 1 & Checklist: Prescriptiveness \\
\hline & Selection of wrong parts/ paths & 1 & Checklist item: Behavioral relation \\
\hline \multirow{3}{*}{$\begin{array}{l}\text { Checklist } \\
\text { Management }\end{array}$} & Difficulty to update & 5 & Checklist: Representation \\
\hline & Selection of wrong checklist & 2 & Checklist: Abstraction, Audience \\
\hline & Difficulty to manage variety of checklists & 2 & Checklist: Scope, Abstraction, and Audience \\
\hline \multirow[t]{5}{*}{$\begin{array}{l}\text { Organizational } \\
\text { Context }\end{array}$} & $\begin{array}{l}\text { False impression work is well done/ tasks are } \\
\text { well understood }\end{array}$ & 9 & Checklist: Prescriptiveness \\
\hline & $\begin{array}{l}\text { Senior staff loathes use/ fears loss of } \\
\text { autonomy }\end{array}$ & 6 & Checklist: Prescriptiveness, Audience \\
\hline & Success depends on implementation & 4 & Checklist: Representation \\
\hline & Does not foster communication/teamwork & 2 & Checklist: Scope \\
\hline & Creates anxiety with subjects (patients) & 2 & Checklist: Abstraction \\
\hline
\end{tabular}

\subsection{Reported Problems with Checklists}

The 21 issue types we identified are summarized in Table 2, along with their citations as a proxy for perceived importance, and their association to the properties of checklists as information artifacts (Table 1). The identified issues are also categorized into four classes:

- Checklist Design: Issues that pertain to the initial development of a checklist for guidance in a specific context;
- Operational Use: Issues that relate to the operational use of checklist that has been designed for a problem domain;

- Checklist Management: Issues that deal with maintaining a checklist or a group of checklists beyond their initial design and initial use;

- Organizational Context: Problems that involve the organizational setting in which a checklist is used.

In Table 2 we further linked the issue types to the checklist properties that we discussed in Section 4. Note that the properties that are most often involved 
in the issues we identified are Representation, Prescriptiveness, and Abstraction.

\subsection{Reported Solutions for Checklist Problems}

We reasoned that papers that would devote considerable attention to describe and characterize structural problems with checklists would also be the most likely sources to discuss solutions proposed or even already applied to deal with these. Using the same pool of papers and once again relying on an iterative style of open coding, we re-analyzed the literature to identify strategies that diminish or eliminate recurring problems associated to the use of checklists as captured in Table 2. We found 14 such strategies in the pool of papers. These strategies can be divided into two categories.

The first category, organizational strategies, covers those strategies that focus on the introduction and uptake of a checklist within its organizational setting:

1. Include the use of a checklist within an organizational improvement cycle, including the pro-vision of feedback as to its actual usage and its effectiveness [1][27][28][29][30][31];

2. Extensively train the staff that is expected to use a checklist prior to its first use [13][27][28] [30][31][32][33][34];

3. Provide insight into the evidence behind the checklist items and clarify the method that has been used to develop the checklist [1][31];

4. Instill accountability for the actual (non)use of a checklist or even enforce its usage [29][31] [33][34][35];

5. Select champions for the use of a checklist and insist on an organization's leadership to promote and support the checklist program [28][31][32][33];

6. Clearly define the different roles and responsibilities that people have in the use of a checklist [13][33];

7. Closely integrate a checklist with the existing systems and operational processes [29][31][32][35][37];

8. Limit the organizational use of checklists to where their application is appropriate [38][39].

The second category of strategies, adaptation strategies, deal with solutions that involve changing the checklist itself:

9. Properly design checklists, in particular to the extent that they are clear and simple [13][29][35][40][41][42];
10. Adapt centrally designed checklists to fit with the local circumstances in which it they are to be applied [27][29][31][33][36];

11. Establish an end-to-end coverage of the checklist, for example: from pre-operative procedures to the actual discharge of a patient [35][30];

12. Use the advantages of electronic checklists [13][35][40][43][44];

13. Similarly design different checklists when they are used by the same staff (to make infrequent use easier) [43];

14. Allow that a checklist can be combined with other modes of process guidance [43].

In the following section, we will discuss the insights we obtained from studying these strategies.

\section{Discussion}

We evaluated the suggested strategies versus the identified problems and arrived at three observations.

Observation 1: State-of-the-art ignores the informational nature of checklists. We found the identified organizational strategies not to be much different from those strategies that are proposed when introducing new concepts or IT systems in organizations [45] or on governance issues with other representation methods for work procedures, say, process models [46]. Similar to these, the checklist strategies focus on sponsorship, training, and adoption, while also calling for a proper reflection on appropriate use. In this sense, we contend that these strategies are not at all checklist-specific. It is striking, though, that the strategies in this category are the ones that receive most attention in the recent literature. As a case in point, all identified publications that advocate organizational strategies are published in the year 2009 or later. Also, the most recent papers that we found emphasize this type of strategy, see [27][30][31][39]. This suggests that the state-of-the-art on overcoming checklist issues does not relate to the nature of the checklist as an informational artifact.

Observation 2: Many important problems are not attended to. We found that the various strategies suggested in the literature indicate an imbalance in how they address the problems that we identified earlier. While some categories of problems are thoroughly covered, the problems in the other categories receive far less attention or none at all. Specifically: 
- All problems in the 'Organizational Context' category are explicitly addressed. Indeed, issues that deal with the lack of adoption of the guidelines by the clinical staff or their inappropriate use of checklists seem to be at the core of many of the organizational strategies that we found, see [28][31].

- The problems in the 'Checklist Design' category are generally considered as solved or avoidable. For example, in publications from the previous century, the development of items to measure progress in an operational process or to guide a user were seen as considerable, see e.g. [47]. In more recent literature, the view is that "figuring out what should form the content of a checklist for a [...] problem is a nonetheless achievable ambition" [32]. Also, there appears to be consensus that checklists should not be applied in all situations, for example when unexpected events are frequent [39]. By contrast, our analysis of the issues in Table 2 suggests, that several design properties of checklists appear to be recurring issues, in particular those in relation to Representation, Prescriptiveness, and Abstraction.

- As to the problems in the 'Operational Use' category, many of the pertinent issues do receive attention - in particular the issues of "checklist fatigue" and non-compliance - yet three notable problems lack any substantial reflection on to how they are to be overcome:

- checklists are not sensitive to context or case [31];

- checklists have no predictive/ prescriptive power [48]; and

- checklists have difficulties in dealing with exceptions [35].

Notably, these three problems all relate to properties of the checklist of an artifact, in terms of its representational, prescriptive and abstraction aspect. The problem of context/case sensitivity is the most cited problem within the 'Operational Use' category. While some solutions specifically aim at adapting a checklist to fit with the local circumstances [29][31], this always relates to the a-priori design of the checklist - not its run-time adaptation. The other two problems have not been addressed at all.

- None of problems in the 'Checklist Management' category have been addressed by any of the solutions. This indicates that a checklist is not recognized as an artifact that has a life cycle of its own and, as such, could be supported by instruments and techniques to manage this life cycle.
From this discussion, we conclude that important problems are left unattended to.

Observation 3: IT solutions have potential. Solution strategy \#12, to use the advantages of an electronic checklist, is, from all the ones identified, the only strategy that picks up on the informational nature of a checklist (i.e. at a deep level structure instead of its physical surface structure [49]). But for some reason this strategy has only been pursued cursorily. Yet, precisely an electronic format for a checklist, in theory, can simultaneously address issues related to aspects of Representation, Scope, Audience and Prescriptiveness. More important, through its digitization, a checklist has the potential to evolve into a "smart machine" cf. [50], in the sense that it can provide abilities not only to inform but also automate work, much like contemporary enterprise IT systems.

To determine the potential of this view, let us reconsider the open problems we detected in the previous subsection (highlighted grey in Table 2). As to the 'Operational Use' category, an electronic checklist, as part of an IT system, could:

- adjust itself depending on the context it is used in or the case it is used for; for example, the logic for behavioral relations between checklist items may only hold under certain conditions, which can be automatically checked; the behavior of the checklist is then adapted without the user having to take any action;

- predict which items may become relevant or critical; this can be done, for example, by automatically collecting previous data on the usage of a checklist and monitoring new instantiations for commonalities with these historic cases;

- adapt the level of leniency on accepting deviations from its idealized execution or change its actual content at run-time if the user signals an exceptional situation.

As to the problems in the 'Checklist Management' category, a digitized checklist would be easier:

- to be remotely updated, similar to how new versions of software versions are electronically distributed;

- to be incorporated in a decision procedure perhaps implemented as a "master checklist" - to determine what the most appropriate checklist is to be used for a specific case;

- to be incorporated as member of a product family of checklists, with facilities for version management, re-use of functionality, configuration, etc. 
All of these strategies admittedly lack much detail at this point, but we hope that the reader can envisage these and agree with us that they lie within the capabilities of modern IT systems. Therefore, strategies and technologies that build on the informational nature of a checklist seem attractive to exploit.

\section{Conclusion}

In this paper, we adopted an informational view on checklists to offer a new conceptualization of these artifacts. In addition, we carried out a thorough analysis of the persistent problems that are associated with the organizational use of checklists. When we analyzed these problems with this informational view in mind, we drew the conclusion that the capabilities of IT systems offer a rich potential to better manage (digital) checklists and put them to operational use.

Our paper can be seen as a call to action for the IT systems community to embrace checklists as appropriate and worthy artifacts of study. Until this point, some may see checklists as trivial artifacts that can be left to be designed by professionals from their various application domains. Given the huge impact that the proper usage of checklists can have on the quality of procedures in settings such as healthcare, manufacturing, aviation, and many more, we feel that IT researchers may be compelled by the impact they can make with joining this research line.

\section{References}

[1] Haynes, A. B. et al. (2009). “A surgical safety checklist to reduce morbidity and mortality in a global population." The New England Journal of Medicine, 360, 491-499.

[2] Hales, B. M. and Pronovost, P. J. (2006). "The checklist: A tool for error management and performance improvement." Journal of Critical Care, 21 (3), 231-235.

[3] Thomassen, Ø. et al. (2010). "The effect of a simple checklist on frequent pre-induction deficiencies." Acta Anaesthesiologica Scandinavica, 54 (10), 1179-1184.

[4] Rosen, D. (2010). "The checklist manifesto: How to get things right." JAMA 303 (7), 670-673.

[5] Winters, B. D., Gurses, A. P., Lehmann, H., Sexton, J. B., Rampersad, C. J. and Pronovost, P. J. (2009). "Clinical review: checklists-translating evidence into practice.” Critical Care, 13(6), 210.

[6] World Health Organization (2009). Surgical Safety Checklist. http://www.who.int/patientsafety/safesurgery/en/

[7] Burton-Jones, A. and Weber, R. (2014). "Building Conceptual Modeling on the Foundation of Ontology." In Computing Handbook, Third Edition:
Information Systems and Information Technology (Topi, H. and Tucker, A., Eds), pp 15-1-15-24, CRC Press, Boca Raton, Florida.

[8] Curtis, B., Kellner, M. I. and Over, J. (1992). "Process Modeling." Communications of the ACM, 35 (9), 75 90.

[9] Recker, J., Rosemann, M., Indulska, M. and Green, P. (2009). "Business Process Modeling: A Comparative Analysis." Journal of the Association for Information Systems, 10 (4), 333-363.

[10] Pentland, B. T. and Rueter, H. H. (1994). "Organizational Routines as Grammars of Action." Administrative Science Quarterly, 39 (3), 484-510.

[11] Schneider, F. B. (1990). "Implementing Fault-Tolerant Services Using the State Machine Approach: a Tutorial." ACM Computing Surveys, 22 (4), 299-319.

[12] Shanks, G., Moody, D. L., Nuredini, J., Tobin, D. and Weber, R. (2010). "Representing Classes of Things and Properties in General in Conceptual Modelling: An Empirical Evaluation." Journal of Database Management, 21 (2), 1-25.

[13] Verdaasdonk, E. G. G., Stassen, L. P. S., Widhiasmara, P. P. and Dankelman, J. (2009). "Requirements for the design and implementation of checklists for surgical processes." Surgical endoscopy, 23 (4), 715-726.

[14] Paull, D. E., Mazzia, L. M., Wood, S. D., Theis, M. S., Robinson, L. D., Carney, B., Neily, J., Mills, P.D. \& Bagian, J. P. (2010). "Briefing guide study: preoperative briefing and postoperative debriefing checklists in the Veterans Health Administration medical team training program." The American Journal of Surgery, 200 (5), 620-623.

[15] Degani, A. and Wiener, E. L. (1990). "Human factors of flight-deck checklists: the normal checklist." Ames Research Center.

[16] Degani, A. and Wiener, E. L. (1993). "Cockpit checklists: Concepts, design, and use." Human Factors: The Journal of the Human Factors and Ergonomics Society, 35 (2), 345-359.

[17] Hilton, B. (2012). "Comparing the Effects of Simulated, Intelligent Audible, Checklists and Analog Checklists in Simulated Flight."

[18] Diamond, T. and Mole, D. J. (2005). "Anatomical orientation and cross-checking - the key to safer laparoscopic cholecystectomy." British journal of surgery, 92 (6), 663-664.

[19] Alter, S. (2014). "Theory of Workarounds." Communications of the Association for Information Systems 34 (55), 1041-1066.

[20] Briere, J. and Runtz, M. (1989). "The Trauma Symptom Checklist (TSC-33). Early data on a new scale.” Journal of Interpersonal Violence, 4 (2), 151163.

[21] Seoane, P. J. G. (2001). "Use and limitations of checklists. Other strategies for audits and inspections." The Quality Assurance Journal, 5 (3), 133-136.

[22] Robins, D. L., Fein, D., Barton, M. L. and Green, J. A. (2001). "The modified checklist for autism in toddlers: An initial study investigating the early detection of autism and pervasive developmental disorders." 
Journal of autism and developmental disorders, 31 (2), 131-144.

[23] Scriven, M. (2005). "The logic and methodology of checklists." http://preval.org/documentos/2075.pdf

[24] Scriven, M. (2007). "Key evaluation checklist (KEC).'

[25] Mauro, R., Degani, A., Loukopoulos, L. and Barshi, I. (2012). "The Operational Context of Procedures and Checklists in Commercial Aviation." In Proceedings of the Human Factors and Ergonomics Society Annual Meeting, 56 (1), 758-762. SAGE Publications.

[26] Paré, G., Trudel, M.-C., Jaana, M. and Kitsiou, S. (2015). "Synthesizing Information Systems Knowledge: A Typology of Literature Reviews." Information \& Management, 52 (2), 183-199.

[27] Anthes, E. (2015). "The Trouble with Checklists." Nature, 523, 516-518.

[28] Conley, D.M. et al. (2011). "Effective surgical safety checklist implementation." Journal of the American College of Surgeons, 212 (5), 873-879.

[29] Fourcade, A. et al. (2011). "Barriers to staff adoption of a surgical safety checklist.” BMJ quality \& safety, 21 (3), 191-197.

[30] Urbach, D. R. et al. (2014). "Introduction of surgical safety checklists in Ontario, Canada." The New England Journal of Medicine, 370 (11), 1029-1038.

[31] Russ, S. J. et al. (2015). "A qualitative evaluation of the barriers and facilitators toward implementation of the WHO surgical safety checklist across hospitals in England: Lessons from the surgical checklist implementation project." Annals of Surgery, 261 (1), 81-91.

[32] Bosk, C. L. et al. (2009). "Reality check for checklists." The Lancet, 374 (9688), 444-445

[33] Vats, A., Vincent, C. A., Nagpal, K., Davies, R. W., Darzi, A. and Moorthy, K. (2010). "Practical challenges of introducing WHO surgical checklist: UK pilot experience." BMJ, 340.

[34] Bliss, L. A. et al. (2012). "Thirty-day outcomes support implementation of a surgical safety checklist." Journal of the American College of Surgeons, 215 (6), 766-776.

[35] de Vries, E.N. et al. (2009). "Development and validation of the SURgical PAtient Safety System (SURPASS) checklist." Quality and Safety in Health Care, 18 (2), 121-126.

[36] Lingard, L. et al. (2011). "Evaluation of a preoperative checklist and team briefing among surgeons, nurses, and anesthesiologists to reduce failures in communication." Archives of Surgery, 143 (1), 12-17.

[37] de Vries, E.N. et al. (2010). "Effect of a comprehensive surgical safety system on patient outcomes." The New England Journal of Medicine, 363 (20), 1928-1937.

[38] Davidoff, F. (2010). "Checklists and guidelines: Imaging techniques for visualizing what to do." JAMA, 304 (2), 206-207.

[39] Stock, C. T. and Sundt, T. (2015). "Timeout for checklists?" Annals of Surgery, 261 (5), 841-842.

[40] Blike, G. and Biddle, C. (2000). "Preanesthesia detection of equipment faults by anesthesia providers at an academic hospital: Comparison of standard practice and a new electronic checklist." AANA Journal, 68 (6), 497-505.

[41] Helmreich, R. L. (2000). "On error management: Lessons from aviation." British Medical Journal, 320 (7237), 781-785.

[42] van Klei, W. A. et al. (2012). "Effects of the introduction of the WHO Surgical Safety Checklist on in-hospital mortality: A cohort study." Annals of Surgery 255 (1), 44-49.

[43] Boorman, D. (2001). "Today's electronic checklists reduce likelihood of crew errors and help prevent mishaps." ICAO Journal, 1, 1-20.

[44] Hart, E. M. and Owen, H. (2005). "Errors and omissions in anesthesia: A pilot study using a pilot's checklist." Anesthesia and Analgesia, 101, 246-250.

[45] Walsham, G. (1993). "IS Strategy and Implementation: A Case Study of a Building Society." SIGOIS Bulletin, 14 (2), 13-16.

[46] Indulska, M., Recker, J., Rosemann, M. and Green, P. (2009). "Process Modeling: Current Issues and Future Challenges." In Advanced Information Systems Engineering - CAiSE 2009 (van Eck, P. and Gordijn, J. and Wieringa, R., Eds), pp 501-514, Springer, Amsterdam, The Netherlands.

[47] Wells, G.L., Leippe, M.R. and Ostrom, T. M. (1979). "Guidelines for empirically assessing the fairness of a lineup." Law and Human Behavior 3 (4), 285-293.

[48] Phelps, D. C. (2004). "Information System Security: Self-Efficacy and Security Effectiveness in Florida Libraries". Electronic Theses, Treatises and Dissertations. Paper 291.

[49] Wand, Y. and Weber, R. (1990). "An Ontological Model of an Information System." IEEE Transactions on Software Engineering, 16 (11), 1282-1292.

[50] Zuboff, S. (1988). In the age of the Smart Machine: The future of work and power. USA: Basic Book. 